\title{
Whole genome sequencing of live attenuated bovine adenovirus type 7 vaccine strain TS-GT reveals biomarkers for virulence attenuation
}

\section{Asuka Kumagai}

National Institute of Animal Health, NARO

\section{Sayo Kajikawa}

Himeji Livestock Hygiene Service Center

\section{Ayako Miyazaki}

National Institute of Animal Health, NARO

Shinichi Hatama ( $\square$ hatama@affrc.go.jp)

National Institute of Animal Health, NARO https://orcid.org/0000-0001-7749-0114

\section{Research Article}

Keywords: Cattle Industry, Respiratory and Enteric Pathogens, Viral Pathogenicity, Next-generation Sequencing, Amino Acid Change, Membrane-lytic Function, Temperature-specific Growth Characteristic

Posted Date: March 11th, 2021

DOl: https://doi.org/10.21203/rs.3.rs-276827/v1

License: (c) (i) This work is licensed under a Creative Commons Attribution 4.0 International License. Read Full License 


\section{Abstract}

Bovine adenovirus type 7 (BAdV-7) is one of the most important respiratory and enteric pathogens in the cattle industry. Although live attenuated vaccines are used to control the virus in Japan, limited information is available on the genomic regions that determine viral pathogenicity. Here we analyzed the attenuated strain TS-GT after isolation of the virus from the hexavalent bovine respiratory disease vaccine Cattlewin-6 lot \#40-1. The complete genome sequence of TS-GT was determined using nextgeneration sequencing. The genome is 30,052 bp long and contains 45 -bp inverted terminal repeats (ITR) and 30 predicted genes. A genome sequence comparison showed that $99.9 \%$ of the TS-GT genome is identical to the prototypic and pathogenic BAdV-7 strain Fukuroi; however, the TS-GT genome contains a novel mutation and four indels. A mutation in TS-GT of the minor capsid protein pVI causes an amino acid change, and possibly induces an alteration of its membrane-lytic function; this may underlie the temperature-specific growth characteristic of this strain. A 19-bp insertion in the 3' ITR may affect its promoter activity and change the expression of downstream coding genes. In this way, the insertion may play important roles in the attenuation of the virus. Although further mutational and functional analyses are required, our results provide insights into the molecular basis of BAdV-7 attenuation and open new avenues for future analyses.

\section{Main Text}

Bovine adenovirus type 7 (BAdV-7) is a double-stranded linear genomic DNA virus that belongs to the genus Atadenovirus (family Adenoviridae) and is one of the most important respiratory and enteric pathogens in the cattle industry [1, 2]. BAdV-7 was first isolated in 1965 in Japan from blood samples of a Holstein cow with respiratory disease $[1,3,4]$. The virus was designated strain Fukuroi and demonstrated to be pathogenic to cattle $[1,5]$. In order to develop a Fukuroi-based live vaccine, serial passaging for 4 to 5 weeks with bovine kidney cells was performed at $30^{\circ} \mathrm{C}$ ( 10 passages in total), followed by short-term passaging at $30^{\circ} \mathrm{C}$ with bovine testicle cells (14 passages), and then with goat testicle (GT) cells (5 passages) [5]. After plaque-purification of the virus (three passages with GT cells), a virus with the characteristic features of temperature-specific growth at $30^{\circ} \mathrm{C}$ and attenuated pathogenicity to cattle was developed; these features led to the TS-GT for the strain [5]. Currently, multivalent live vaccines containing TS-GT are available in Japan (https://www.kyotobiken.co.jp/en/products/cow.html\#respiration), but little is known about the genomic characteristics of the virus.

Here we isolated BAdV-7 strain TS-GT from the hexavalent bovine respiratory disease vaccine Cattlewin-6 lot \#40-1 (Kyoto Biken Laboratories Inc.). The vaccine was treated with $10 \%$ chloroform at $20^{\circ} \mathrm{C}$ for 30 min to inactivate other constituent viruses, namely, bovine infectious rhinotracheitis virus, bovine viral diarrhea virus type 1 and 2, bovine respiratory syncytial virus, and bovine parainfluenza 3 virus. The suspension was then serially diluted and mixed with bovine embryonic testicle (BET) cells. Supernatants from the end-point dilutions showing cytopathic effects were collected. The isolate was confirmed to be 
positive for BAdV-7 and negative for the other five bovine viruses by PCR; sequencing indicated that the strain was TS-GT $[6,7]$. The virus was expanded by large-scale-culture with BET cells, followed by centrifugation to remove cellular debris. The stocks were semi-purified by discontinuous sucrose density gradient ultra-centrifugation and dissolved in phosphate-buffered saline. DNA was extracted using a QIAamp MinElute Virus Spin Kit (Qiagen) and submitted to Macrogen Japan Co. Ltd.

(https://www.macrogen-japan.co.jp/) for whole-genome sequencing. Briefly, sequence libraries were constructed using a TruSeq Nano DNA sample preparation kit (lllumina). DNA sequencing was performed with a deep sequencing protocol using Novaseq 6000 (Illumina). A total of 45 million paired-end reads (a total of 6.8 billion bases) were obtained with an average length of $151 \mathrm{bp}$. Bases with a phred quality score below 20 were trimmed from every read and the reads were assembled using a de novo approach with Trimmomatic version 0.36 (http://www.usadellab.org/cms/?page=trimmomatic) and SPAdes version 3.13 .0 (http://cab.spbu.ru/software/spades/) with default settings. A 29,941 bp contig was generated with an average base coverage depth of 217. To account for short nucleotide stretches lacking at both ends of the genome, $5^{\prime}$ and $3^{\prime}$ adapter ligation, followed by PCR with specific primers, and Sanger sequencing was performed [8]. A full-length genome sequence of TS-GT of 30,052 nucleotides with $33.58 \%$ GC content was obtained. Inverted terminal repeat (ITR) sequences of 45 bp were present. Putative open reading frames and functions of the translated products were predicted using the DNA data bank of Japan (DDBJ) fast annotation and submission tool (https://dfast.nig.ac.jp/). The obtained sequence encoded 30 predicted genes.

Compared with the complete sequence of Fukuroi (GenBank accession number LC597488), TS-GT had a nucleotide mutation (at 14,234 nt), two deletions (at $14 \mathrm{nt}$ and 30,021 nt), and two insertions (at 13,685 nt and 29,999 nt) (Table 1). The 14,234 nt mutation is located in the central region of the minor capsid pVI protein and is expected to induce an amino acid change from lysine 96 to asparagine (Fig. 1A). The amino acid change may affect its biological function. It has been reported that adenovirus $\mathrm{pVI}$ is a multifunctional protein (its best-known function is disruption of the endosomal membrane) that is crucial for decapsidation and release from the endosome in the early phase of infection [9]. It has also been reported that temperature-sensitive (ts) mutants of human adenovirus type 5 express a very stable form of the $\mathrm{pVI}$ proteins in the endosomal environment and that ts mutants significantly reduce membrane lytic activities compared with wild-type viruses [10]. This behavior underlies the characteristic ts biotype. On the basis of previous reports, the mutation in pVI is likely to play an important role in the ts dependent growth of TS-GT. The deletions at $14 \mathrm{nt}$ and 30,021 nt are located in the core origins of the $5^{\prime}$ and $3^{\prime}$ ITR promoter regions (Fig. 1B and 1C). Zhu et al. reported that similar deletions are found in the ITR of BAdV type 3 (BAdV-3) strain HLJ0955 compared with the prototype BAdV-3 strain WBR-1 [11]. Indels in the ITR regions seems to be common in BAdV. Whether or not these nucleotide changes affect adenoviral promoter function is still controversial and needs further investigations [12, 13]. The insertion at 13,679 nt is located in the non-coding region between $\mathrm{pVII}$ and $\mathrm{pX}$ and does not change the protein-coding sequence (Table 1). Interestingly, the ITRs of TS-GT, at both ends of the genome, were nine bp longer than in Fukuroi (Fig. 1B and 1C). Notable changes were found in the 3' ITR region. Next to an SP1transcription-factor binding site, a 19-bp DNA fragment is inserted in the TS-GT genome but not in 
Fukuroi (Fig. 1C). This region is thought to regulate the 3' ITR promoter activity. The region downstream of the 3' ITR promoter encodes five predicted proteins of unknown function, $\mathrm{RH} 1$ to $\mathrm{RH}$, and three $\mathrm{E} 4$ proteins, E4.1 to E4.3 that are key viral and cellular regulatory factors involved in transcription, apoptosis, cell cycle control, and repair [14]. It is possible that these genes function as trans-acting regulators by modulating host immune responses to viral infection and are involved in attenuation. In some adenoviruses, e.g., egg drop syndrome virus, virus-associated RNA (VA RNA) is also encoded in the RH region [15]. VA RNAs are non-protein coding sequences that can antagonize the antiviral activity of interferons [16]. However, in our study, we did not identify any sequences with homology to VA RNA in the corresponding region of the TS-GT genome. Although further mutational and functional analyses are required to clarify the effect of these mutation and indels in the TS-GT genome, our results provide insights into the molecular basis of BAdV-7 attenuation and open new avenues for future analyses.

\section{Declaration}

Data availability The raw read data and the complete genome sequence of TS-GT have been deposited in DDBJ under accession nos. DRR270494 and LC606503.

Funding This study received no specific grants from any funding agency in the public, commercial, or notfor-profit sectors.

\section{Compliance with ethical standards}

Conflict of interest The authors declare that they have no conflicts of interest.

Ethical approval This article does not contain any studies with live animals performed by any of the authors.

Consent to participate and publication All authors confirm that they have read the manuscript and participated in the study.

\section{References}

1. Inaba Y, Tanaka Y, Stao K et al (1968) Bovine adenovirus II. A serotype, Fukuroi, recovered from Japanese cattle. Jpn J Microbiol 12:219-229

2. Härtel H, Nikunen S, Neuvonen E et al (2004) Viral and bacterial pathogens in bovine respiratory disease in Finland. Acta Vet Scand. 45:193-200

3. Matumoto M, Inaba Y, Tanaka Y et al (1969) Serological typing of bovine adenovirus, Nagano and Fukuroi, as type 4 and new type 6. Jpn J Microbiol 13:131-132

4. Matumoto M, Inaba Y, Tanaka Y et al (1970) New serotype 7 of bovine adenovirus. Jpn J Microbiol 14:430-431 
5. Inaba Y, Murase N, Kumagai T et al (1976) Bovine adenovirus type 7 live vaccine. In: Studies on control of respiratory and enteric infectious diseases in calves (AgriKnowledge 87). Published by Tsukuba Business-Academia Cooperation Support Center, Agriculture, Forestry and Fisheries Research Council Secretariat, pp 46-51. https://agriknowledge.affrc.go.jp/RN/2039014270.pdf (in Japanese)

6. Toker EB, Yeşilbağ K. (2021) Molecular characterization and comparison of diagnostic methods for bovine respiratory viruses (BPIV-3, BRSV, BVDV, and BoHV-1) in field samples in northwestern Turkey. Trop Anim Health Prod. 53:79

7. Maluquer de Motes C, Clemente-Casares P, Hundesa A et al (2004) Detection of bovine and porcine adenoviruses for tracing the source of fecal contamination. Appl Environ Microbiol. 70:1448-1454

8. Kumagai A, Kajikawa S, Miyazaki A et al (2021) Complete genome sequence of bovine adenovirus type 7 strain Fukuroi from a cow with respiratory disease. Microbiol Resour Announc. in press

9. Hernando-Pérez M, Martín-González N, Pérez-Illana M et al (2020) Dynamic competition for hexon binding between core protein VII and lytic protein VI promotes adenovirus maturation and entry. Proc Natl Acad Sci USA. 117:13699-13707

10. Wiethoff $\mathrm{CM}$, Wodrich $\mathrm{H}$, Gerace $\mathrm{L}$ et al (2005) Adenovirus protein $\mathrm{VI}$ mediates membrane disruption following capsid disassembly. J Virol. 79:1992-2000

11. Zhu YM, Yu Z, Cai H et al (2011) Isolation, identification, and complete genome sequence of a bovine adenovirus type 3 from cattle in China. Virol J. 8:557

12. van Olphen AL, Mittal SK. (2002) A 72-bp internal deletion in the left inverted terminal repeat of the bovine adenovirus type 3 genome does not affect virus replication. Intervirology. 45:188-192

13. Wunderlich K, van der Helm E, Spek D et al (2014) An alternative to the adenovirus inverted terminal repeat sequence increases the viral genome replication rate and provides a selective advantage in vitro. J Gen Virol. 95:1574-1584

14. Weitzman MD. (2005) Functions of the adenovirus E4 proteins and their impact on viral vectors. Front Biosci. 10:1106-1117

15. Hess M, Blöcker H, Brandt P. (1997) The complete nucleotide sequence of the egg drop syndrome virus: an intermediate between mastadenoviruses and aviadenoviruses. Virology. 238:145-156

16. Mathews MB, Shenk T. (1991) Adenovirus virus-associated RNA and translation control. J Virol. 65:5657-5662

\section{Tables}

Table 1 is available in the Supplementary Files.

\section{Figures}


A

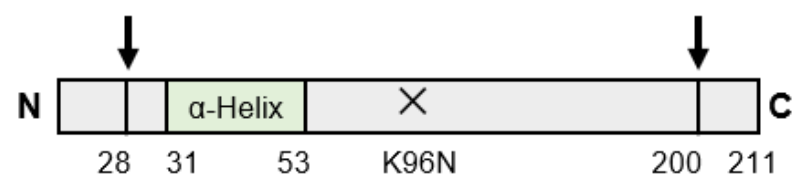

B

14

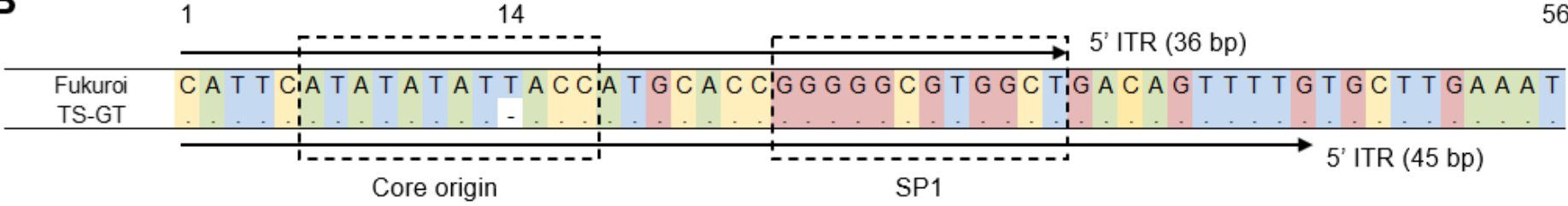

C

30,021

30,034

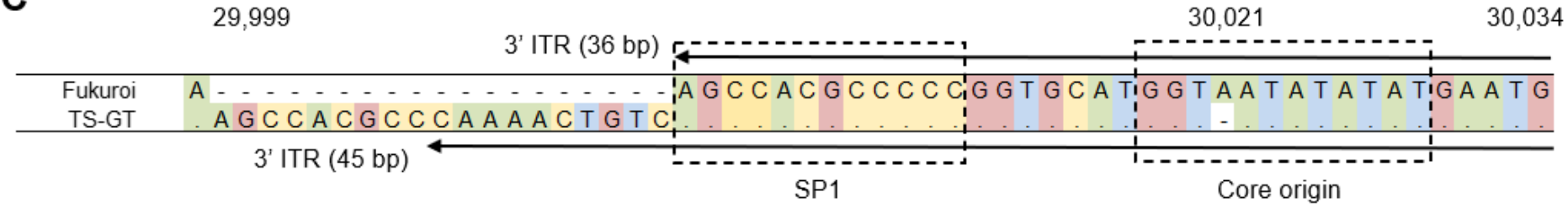

\section{Figure 1}

(A) Schematic representation of the pVI protein. The green box shows the a-helix domain. Proteolytic cleavage sites in both the $\mathrm{N}$ and $\mathrm{C}$ terminal regions are indicated by vertical arrows. Numbers show the amino-acid positions of the pVI protein. X represents the mutation point. Alignment of the (B) 5' and (C) 3' terminal sequences of Fukuroi and TS-GT. Dotted boxes show the core origin and the SP1 binding site. Horizontal arrows above and below the alignment indicate the ITR regions of Fukuroi and TS-GT. Numbers represent nucleotide positions in the Fukuroi genome.

\section{Supplementary Files}

This is a list of supplementary files associated with this preprint. Click to download.

- Table1Kumagaietal.xlsx 